\title{
Formulation of 2D Graphene Deformation Based on Chiral-Tube Base Vectors
}

\author{
Bohua Sun \\ Department of Mechanical Engineering, Cape Peninsula University of Technology, P.O. Box 1906, Bellville 7535, \\ City of Cape Town, South Africa
}

Correspondence should be addressed to Bohua Sun, sunb@cput.ac.za

Received 12 May 2010; Accepted 13 June 2010

Academic Editor: Rakesh Joshi

Copyright ( 12010 Bohua Sun. This is an open access article distributed under the Creative Commons Attribution License, which permits unrestricted use, distribution, and reproduction in any medium, provided the original work is properly cited.

The intrinsic feature of graphene honeycomb lattice is defined by its chiral index $(n, m)$, which can be taken into account when using molecular dynamics. However, how to introduce the index into the continuum model of graphene is still an open problem. The present manuscript adopts the continuum shell model with single director to describe the mechanical behaviors of graphene. In order to consider the intrinsic features of the graphene honeycomb lattice-chiral index $(n, m)$, the chiral-tube vectors of graphene in real space have been used for construction of reference unit base vectors of the shell model; therefore, the formulations will contain the chiral index automatically, or in an explicit form in physical components. The results are quite useful for future studies of graphene mechanics.

\section{Introduction}

A one-atom-thick layer of graphite called graphene is the "mother" of all graphitic forms. Graphene is a 2D building material for carbon materials of all other dimensionalities. It can be wrapped up to $0 \mathrm{D}$ buckyballs, rolled into $1 \mathrm{D}$ nanotubes, or stacked into $3 \mathrm{D}$ graphite [1-3]. Figure 1 is a famous picture take on from [3].

The graphene has attracted attention because of its unusual two-dimensional structure and potential for applications. Owing to its exceptional mechanical properties and low mass density, graphene is an ideal material for use in nanoelectromechanical systems (NEMS), which are of great interest both for fundamental studies of mechanics at the nanoscale and for a variety of applications, including force, position, mass, and gas sensing [4].

Graphene research has developed at a truly relentless pace. Several papers appear every day; comprehensive reviews have been done by the graphene pioneers [2, $3]$. Geometrically, the graphene can be considered as an "almost" 2D material due to its very small thickness at only one-atom. The 2D feature of the graphene makes it possible to directly use well-formulated 2D continuum shell model to simulate its deformation, vibration, and buckling [1-3].
Although many features of the graphene can be described by relativistic quantum mechanics and molecular dynamics, the continuum modeling is still useful and crucial for rational device design and interpretation of experimental results. Because experiments at the nanoscale are extremely difficult and atomistic modelling remains prohibitively expensive for large-sized atomic system, it has been accepted that continuum models will continue to play an essential role in the study of carbon nanotube [5]. The validity of using continuum models for nanotube and graphene has been supported by both experimental results and molecular-dynamics simulation, all previous investigations on nanotube have indicated that the laws of continuum mechanics are still valid to some extent even in nanoscale. The success of continuum models to nanotube gives us confidence to predict that contunuum modelling is going to be also valid for graphene mechanics analysis $[6,7]$.

Within the frame of classic continuum mechanics, there is a well-formulated branch-theory of shell [8-14]. It deals with a special $3 \mathrm{D}$ continuum shell; its thickness is very much smaller than its width and length. The deformation of the thinner 3D shell can be considered as a 2D middle surface with a small thickness, and all off-middle surface quantities can be presented in terms of middle surface. In this way, the 


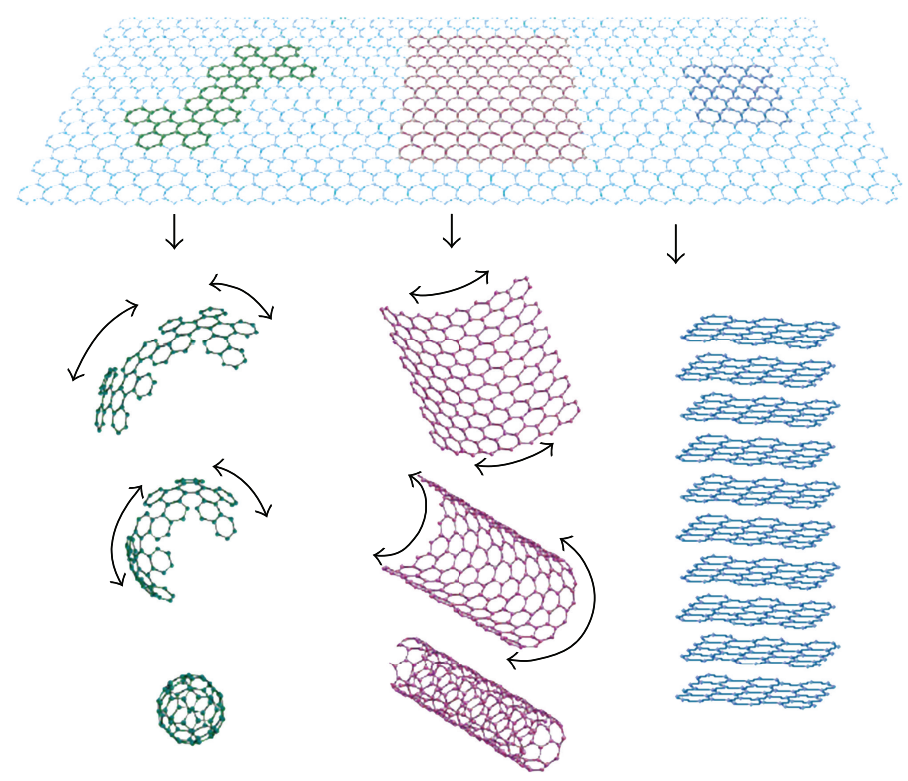

FIGURE 1: Mother of all graphitic forms. Graphene is a 2D building material for carbon materials of all other dimensionalities. It can be wrapped up into $0 \mathrm{D}$ buckyballs, rolled into $1 \mathrm{D}$ nanotubes or stacked into 3D graphite.

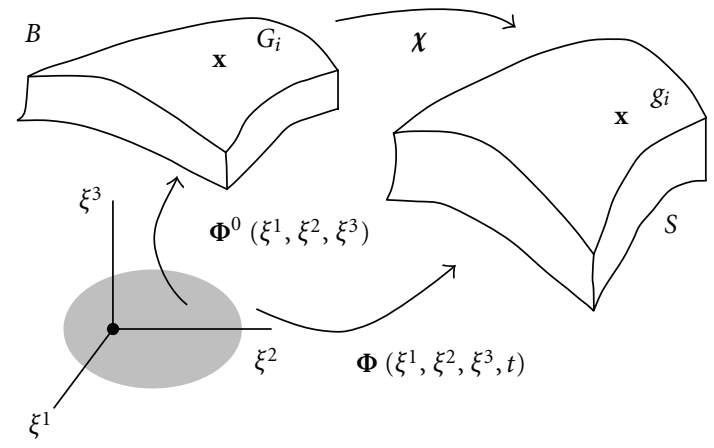

FIGURE 2: Paramterization of the reference and current configurations $B$ and $S$; the departion mapping and basis vectors.

$3 \mathrm{D}$ thinner shell problem is being converted into a $2 \mathrm{D}$ surface problem with a small thickness. Based on this understanding, it is to believe that the shell theory should be the best continuum mechanics model for graphene mechanics.

Regarding the mechanics modeling of the graphene, most of literature considered the graphene as a $2 \mathrm{D}$ prestretched membrane with zero thickness [15-18], and some considered the graphene bending properties [19-22]. Since monolayer graphene is very thin, to some extent the membrane theory could be very simple and quite good estimate model, but for tiny and delicated NEMS devices, any small thing must be taken into account as it might has a significant impact on graphene, for instance, graphene's thickness $[3,6]$.

Although graphene's thickness is very small, one layer graphene thickness is $0.335 \mathrm{~nm}$ [5], but it cannot be ignored as zero, because the thickness plays a key role to supply a bending stiffness. The graphene should be modelled as a very thinner 3D object by shell theory [8-14].
The current shell theory [8-14] can be directly applied to the deformation analysis of nanostructures, for instance, $[7,22]$. Reference [22] considered the effects of surface tension on the elastic properties of nanostructures, and [7] discussed a promised nanomaterials nanotorus. However, how to introduce the chiral index $(n, m)$ into continuum model of the graphene is still an open problem. The present manuscript adopts the continuum shell model with single director to describe the mechanical behaviors of graphene. In order to consider the intrinsic features of the graphene honeycomb lattice-chiral index $(n, m)$, the chiraltube vectors of graphene in real space have been used for construction of reference unit base vectors of the shell model; therefore, the formulations will contain the chiral index automatically, or in explicit form in physical components. The results are quite useful for future studies of graphene mechanics.

The paper is organized as follows. Some elasticity concepts and kinematics of a deformable surface will be introduced in Section 2; the parameterization of single director shell and deformation mode will be demonstrated in Sections 3 and 4. The basis vectors will be established in Section 5. The deformation gradient for both with and without predeformation will be formulated in Sections 6 and 7. The explicit form of the chiral index $(n, m)$ in the physical components will be shown in the Section 8 .

\section{Some Elasticity Concepts}

2.1. Configuration, Deformation, and Parametrization. Let $B$ represent the stress-free or reference placement of the physical body with boundary $\partial B$. Points of $B$ are indentified by $\mathbf{X} \in B$. Let $B$ be parametrized by the one to one point 
mapping function $\boldsymbol{\Phi}$, such that $B=\left\{\mathbf{X}=\boldsymbol{\Phi}\left(\xi^{1}, \xi^{2}, \xi^{3}\right) \in\right.$ $\left.R^{3} \mid\left(\xi^{1}, \xi^{2}, \xi^{3}\right) \in \Omega\right\}$ where $\Omega \subset R^{3} 2$ is the parametric image of the body under the inverse mapping $\Phi^{-1}$. Denote the deformed configuration of the body by $S$ with the boundary of $S$ given by $\partial S$. Points in $S$ are identified by $\mathbf{x} \in S$. The time-dependent configurations are parametrized by one to one point mapping function $\boldsymbol{\Phi}$, such that $S=\{\mathbf{x}=$ $\Phi\left(\xi^{1}, \xi^{2}, \xi^{3}, t\right) \in R^{3}\left|\left(\xi^{1}, \xi^{2}, \xi^{3}\right) \in \Omega, t \in[0, T], \mathbf{x}\right|_{\partial_{d} S}=$ $\overline{\mathbf{x}}\}$ where $\left.\boldsymbol{\Phi}\left(\xi^{1}, \xi^{2}, \xi^{3}, t\right)\right|_{t=0} \equiv \boldsymbol{\Phi}^{0}\left(\xi^{1}, \xi^{2}, \xi^{3}\right)$ and $\overline{\mathbf{x}}$ is the prescribed placement of points on the boundary $\partial_{d} S$ where the placement is given. The deformation mapping $\chi: \mathbf{X} \in$ $B \rightarrow \mathbf{x} \in S$ is given parametrically by $\chi(\mathbf{X}, t)=\mathbf{\Phi}$ 。 $\Phi^{0^{-1}}$. Note that $\chi(\mathbf{X}, 0)=\mathbf{1}$ is the identity mapping.

2.2. Tangent Basis Vectors and the Deformation Gradient. By differentiating the mapping function $\mathbf{x}$ with respect to curvilinear coordinate (convected coordinate) function $\xi^{i}(i=1,2,3)$, the tangent basis vectors are obtained $\mathbf{g}_{i}\left(\xi^{1}, \xi^{2}, \xi^{3}, t\right)=\mathbf{x},_{i}\left(\xi^{1}, \xi^{2}, \xi^{3}, t\right)$. The fact that the tangent basis vectors are linearly independent is expressed by the relationship $\mathbf{g}_{1} \cdot\left(\mathbf{g}_{2} \times \mathbf{g}_{3}\right)>0$. The reference or undeformed tangent basis vectors $\mathbf{G}_{i}$ are obtained by particularizing the current basis vectors to $t=0$, hence $\mathbf{G}_{i}=\mathbf{X}_{i}\left(\xi^{1}, \xi^{2}, \xi^{3}\right)$. The dual basis vectors $\mathbf{g}^{i}$ and $\mathbf{G}^{i}$ are defined by the orthogonality condition $\mathbf{g}^{i} \cdot \mathbf{g}_{j}=\delta_{j}^{i}, \mathbf{G}^{i} \cdot \mathbf{G}_{j}=\delta_{j}^{i}$. The deformation gradient is the mapping $\mathbf{F}: R^{3} \rightarrow R^{3}$ and is obtained by taking the material gradient of the deformation mapping $\chi$ as $\mathrm{F}=T \chi \in$ $\mathrm{GL}_{+}(3)$, where $\mathrm{GL}_{+}(3)=\left\{\mathbf{F} \in R^{3 \times 3} \mid \operatorname{det}(\mathbf{F})>0\right\}$. In terms of the parametrization, $\mathbf{F}$ is given by $\mathbf{F}=T \chi=\Delta \mathbf{x} \Delta \mathbf{X}^{-1}$. Using the above relations, the deformation gradient $F$ can be given in terms of the current tangent basis vectors and the reference dual basis vectors by $\mathbf{F}=\mathbf{g}_{i} \otimes \mathbf{G}^{i}$ or in a coordinate format as $\mathbf{F}=F_{J}^{i} \mathbf{g}_{i} \otimes \mathbf{G}^{J}$, where $F_{J}^{i}=\partial x^{i} / \partial X^{J}$, with relations $\mathbf{F}^{T}=\mathbf{G}^{i} \otimes \mathbf{g}_{i}, \mathbf{F}^{-1}=\mathbf{G}_{i} \otimes \mathbf{g}^{i}$, and $\mathbf{F}^{-T}=\mathbf{g}^{i} \otimes \mathbf{G}_{i}$. Then, the determinant of the deformation gradient $J$ is given by $J=\operatorname{det} \mathbf{F}=j / j^{0}, j=\operatorname{det} \Delta \mathbf{x}=\mathbf{g}_{1} \cdot\left(\mathbf{g}_{2} \times \mathbf{g}_{3}\right), j^{0}=\operatorname{det} \Delta \mathbf{X}=$ $\mathbf{G}_{1} \cdot\left(\mathbf{G}_{2} \times \mathbf{G}_{3}\right)$. Since the current and reference tangent basis vectors are linearly independent, so have $j^{0}>0, j>0, J>0$.

\section{Parameterization of Graphene Shell Model}

The variable pair $(\mathbf{r}, \mathbf{d})$ is chosen normally as kinematic variable, in which $\mathbf{r}$ is the position vector and $\mathbf{d}$ is the director field of mid-surface. Since they are vectors, so the calculations corresponding to these quantities also operate in vector space.

The reasonable parameterization of director field plays a central role in development of shell models. The director field is decomposed into a positive magnitude parameter $\lambda$ and the inextensible component or unit director field $t$, $\mathbf{d}=\lambda \mathbf{t}$, where $\lambda=|\mathbf{d}|, \mathbf{t} \cdot \mathbf{t}=1, t=\mathbf{d} / \lambda$. The unit director field $\mathbf{t}$ is parameterized by two independent angles, that is, $\mathbf{t}=\mathbf{t}\left(\psi_{1}, \psi_{2}\right)=\left\{\sin \psi_{1} \cos \psi_{2} \sin \psi_{1} \sin \psi_{2} \cos \psi_{1}\right\}$. The advantage of this kind of representation of $\mathbf{t}$ is that the variational calculation is very simple, for instance, $\delta \mathbf{t}=$ $\mathbf{t}_{1} \delta \psi_{1}+\mathbf{t}_{2} \delta \psi_{2}$.

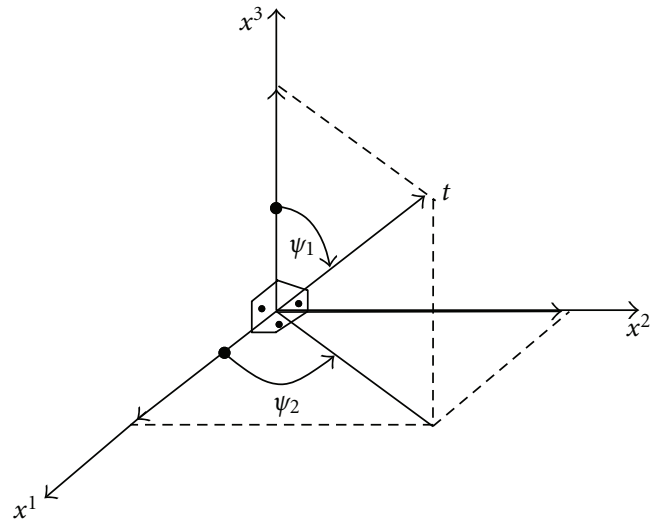

FIGURE 3: Parameterization of single director.

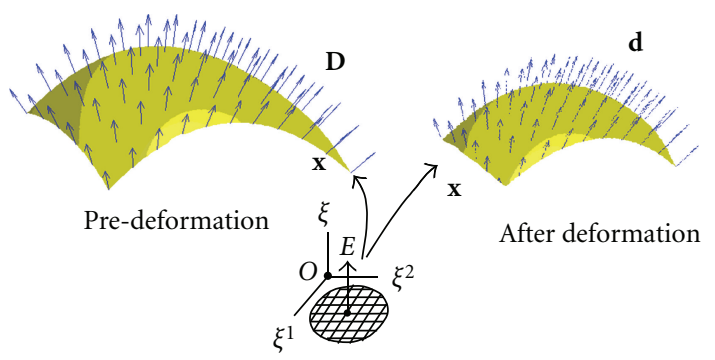

FIgURE 4: Single director shell model middle surface and director field.

\section{Single Director Kinematic Modelling of Graphene}

The variable pair $(\mathbf{r}, \mathbf{d})$ is chosen normally as kinematic variable, in which $\mathbf{r}$ is the position vector and $\mathbf{d}$ is the director field of deforemed mid-surface and denoting $\mathbf{R}$ and $\mathbf{D}$ being the position vector and single director on the middle surface of undeformed configuration.

According to the long tradition of shell research [8$14]$, the single director field in reference or undeformed configuration can be written as

$$
\mathbf{X}=\mathbf{R}\left(\xi^{1}, \xi^{2}, t\right)+\xi \mathbf{D}\left(\xi^{1}, \xi^{2}, t\right) .
$$

The single director field in current configuration can be written as

$$
\mathbf{x}=\mathbf{r}\left(\xi^{1}, \xi^{2}, t\right)+\xi \mathbf{d}\left(\xi^{1}, \xi^{2}, t\right) .
$$

Thus, the points in the body are indentified as the midsurface mapping $\mathbf{r}$ or $\mathbf{R}$ plus the distance $\xi$ along the director d or $\mathbf{D}$.

The deformation mode of shell is such that points intially defined along straight fibers, that is, indentified as the distance $\xi$ above or below the mid-surface along the line defined by the initial director $\mathbf{D}$, remain a straight fibers. From the physical point of view, the solution to the shell problem, under the single director kinematic assumption, can be considered as the solution for the shell mid-surface plus the solution for the director which orients points along thickness fibers. 
Membrane
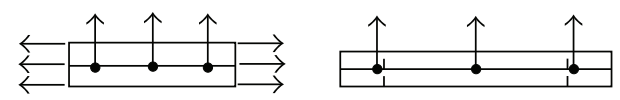

Bending
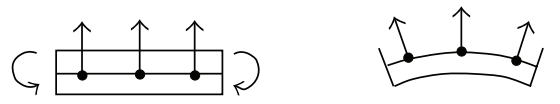

Transverse shear
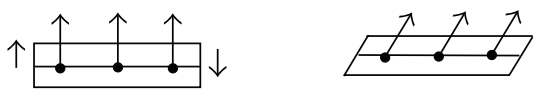

Thickness deformation
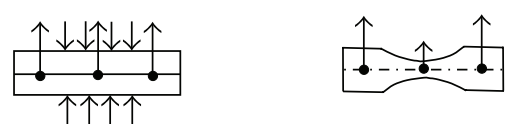

Figure 5: Deformation mode of the single director shell model.

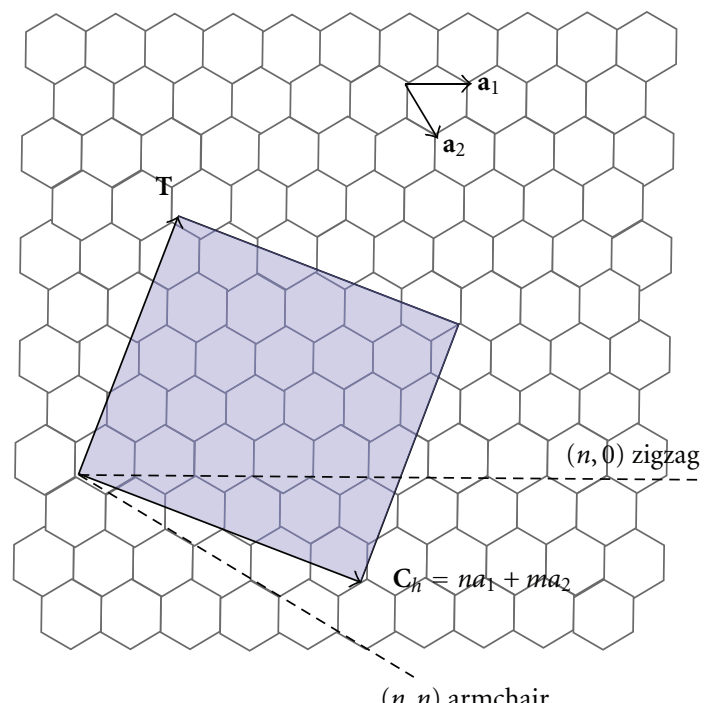

Figure 6: The $(n, m)$ nanotube naming scheme can be thought of as a vector $\left(\mathbf{C}_{h}\right)$ in an infinite graphene sheet that describes how to "roll up" the graphene sheet to make the nanotube. $\mathrm{T}$ denotes the tube axis, and $\mathbf{a}_{1}$ and $\mathbf{a}_{2}$ are the unit vectors of graphene in real space. http://en.wikipedia.org/wiki/Carbon_nanotube.

Let us now check, from geometric understanding, whether the single director kinematic assumption could capture the main characteristics of shell deformation. Finite membrane stretch is captured by the deformation of the mid-surface map $\mathbf{r}$; finite bending strains are modeled by the spatial gradient of the director $\mathbf{d}$; transverse shear strain is accounted for by measuring the relative rotation of the director $\mathbf{d}$ with respect to the normal to the mid-surface. The thickness change can be represented by the difference of magnitude of initial and current director.

\section{Establishment of Unit Base Vectors in the Reference State (Undeformed Graphene Sheet)}

The way the graphene sheet is wrapped is represented by a pair of indices $(n, m)$ called the chiral vector. The integers $n$ and $m$ denote the number of unit vectors along two directions in the honeycomb crystal lattice of graphene. If $m=0$, the nanotubes are called "zigzag". If $n=m$, the nanotubes are called "armchair". Otherwise, they are called "chiral".

The orthogonal base vectors in the reference state of the grapheme can defined as follows: let $\mathbf{a}_{1}$ be a base vector $\mathbf{e}_{1}$, normal base vector of the grapheme plane is $\mathbf{e}_{3}=\mathbf{a}_{1} \times \mathbf{a}_{2} / \mid \mathbf{a}_{1} \times$ $\mathbf{a}_{2} \mid=(2 / \sqrt{3})\left(\mathbf{a}_{1} \times \mathbf{a}_{2}\right)$, and then $\mathbf{e}_{2}=(2 / \sqrt{3}) \mathbf{a}_{2}-(1 / \sqrt{3}) \mathbf{a}_{1}$. The chiral vector $\mathbf{C}_{h}$ can be expressed as follows in terms of orthogonal base vectors: $\mathbf{C}_{h}=(n+(\sqrt{3} / 2) m) \mathbf{e}_{1}+(\sqrt{3} / 2) m \mathbf{e}_{2}$. The tube axes vector $\mathbf{T}$ is orthogonal to the chiral vectors, that is, $\mathbf{T} \cdot \mathbf{C}_{h}=0$, then we have $\mathbf{T}=(\sqrt{3} / 2) m \mathbf{e}_{1}-(n+$ $(\sqrt{3} / 2) m) \mathbf{e}_{2}$.The no-orthogonal base vectors $\left\{\mathbf{a}_{1}, \mathbf{a}_{2}, \mathbf{e}_{3}\right\}$ can be represented by orthogonal base vectors $\left\{\mathbf{e}_{1}, \mathbf{e}_{2}, \mathbf{e}_{3}\right\}$.

Since $\mathbf{T}$ and $\mathbf{C}_{h}$ are orthogonal each other, we can form another chiral-tube base vectors $\left\{\mathbf{C}_{h}, \mathbf{T}, \mathbf{E}_{3}\right\}$, where the normal vector or single director field in undeformed grapheme plane is $\mathbf{E}=\mathbf{C}_{h} \times \mathbf{T}=\left(n^{2}+m \sqrt{3}\right) \mathbf{e}_{3}$.

Up to now, from unit vectors of grapheme in real space, we have formulated two base vectors $\left\{\mathbf{e}_{1}, \mathbf{e}_{2}, \mathbf{e}_{3}\right\}$ and $\left\{\mathbf{C}_{h}, \mathbf{T}, \mathbf{E}\right\}$. In order to capture more information about the deformed grapheme sheet, we will try to formulate its shell model based on the chiral-tube base vectors $\left\{\mathbf{C}_{h}, \mathbf{T}, \mathbf{E}\right\}$; this work has not been found in the literature. For tensor calculation, we introduce $\mathbf{E}^{i}$ to represent the chiral-tube base vectors as follows:

$$
\mathbf{E}^{1}=\mathbf{C}_{h}, \quad \mathbf{E}^{2}=\mathbf{T}, \quad \mathbf{E}^{3}=\mathbf{E} .
$$

Corresponding to the chiral-tube unit base vectors $\left\{\mathbf{C}_{h}, \mathbf{T}, \mathbf{E}\right\}$, we can define three axes $\xi^{i}(i=1,2,3)$, for instance, the any point in the graphene plane can be expressed as follows: $\xi^{1} \mathbf{C}_{h}+\xi^{2} \mathbf{T}=\xi^{1} \mathbf{E}^{1}+\xi^{2} \mathbf{E}^{2}$ and any point out of the graphene plane is $\xi^{2}=\xi^{1} \mathbf{E}^{1}+\xi^{2} \mathbf{E}^{2}+\xi \mathbf{E}$.

\section{Tangent Basis and Deformation Gradient of Graphene without Predeformation}

If the grapheme sheet has no any predeformation, it will be like a plane. Since $\mathbf{E}_{\alpha}=\partial \mathbf{E} / \partial \xi^{\alpha}=0$, we have the tangent basis vectors in the undeformed configuration as follows:

$$
\mathbf{G}_{\alpha}=\mathbf{E}_{\alpha}, \quad \mathbf{G}_{3}=\mathbf{E}_{3} .
$$

After the deformation, the convected tangent basis vectors in current graphene shell configuration is defined by

$$
\mathbf{g}_{\alpha}=\mathbf{x},_{\alpha}=\mathbf{r}_{\alpha}+\xi \mathbf{d}_{,_{\alpha}}, \quad \mathbf{g}_{3}=\mathbf{x},_{3}=\mathbf{d} .
$$

The dual basis vectors are also defined by the relationships $\mathbf{g}^{i} \cdot \mathbf{g}_{j}=\delta_{j}^{i}$ and $\mathbf{E}^{I} \cdot \mathbf{E}_{J}=\delta_{J}^{I}$.

Then, tangent of deformation or deformation gradient $\mathbf{F}$ can be defined by

$$
\mathbf{F}=\mathbf{g}_{i} \otimes \mathbf{G}^{i}=\mathbf{r}_{\alpha} \otimes \mathbf{E}^{\alpha}+\mathbf{d} \otimes \mathbf{E}^{3}+\xi \mathbf{d}_{\alpha} \otimes \mathbf{E}^{\alpha} .
$$


If we put $\xi=0$ in (5), then the basis vectors of the midsurface in the current configuration can be obtained; $\mathbf{a}_{i}=$ $\left.\mathbf{g}_{i}\right|_{\xi=0}$ that is,

$$
\mathbf{a}_{\alpha}=\mathbf{r}_{\alpha}, \quad \mathbf{a}_{3}=\mathbf{d}
$$

and the corresponding dual basis vectors in the mid-surface are defined by $\mathbf{a}^{i} \cdot \mathbf{a}_{j}=\delta_{j}^{i}$.

\section{Deformation Gradient of Graphene with Pre-Deformation}

If $2 \mathrm{D}$ graphene is being wrapped up to $0 \mathrm{D}$ nanodot, $1 \mathrm{D}$ nanotube, 3D stacked graphite, or been prestressed and then undergo deformation, it means that the graphene got predeformation.

After the pre-deformation, the convected tangent basis vectors in predeformation configuration are defined by

$$
\overline{\mathbf{G}_{\alpha}}=\mathbf{X}_{,_{\alpha}}=\mathbf{R}_{\alpha}+\xi \mathbf{D}_{\alpha}, \quad \overline{\mathbf{G}}_{3}=\mathbf{X},_{3}=\mathbf{D} .
$$

The dual basis vectors are also defined by the relationships $\overline{\mathbf{G}}^{i} \cdot \overline{\mathbf{G}}_{j}=\delta_{j}^{i}$.

The predeformation gradient $\mathbf{F}_{\text {prewill be }}$

$$
\mathbf{F}_{\text {pre }}=\overline{\mathbf{G}}_{i} \otimes \mathbf{G}^{i}=\mathbf{R}_{\alpha} \otimes \mathbf{E}^{\alpha}+\mathbf{D} \otimes \mathbf{E}^{3}+\xi \mathbf{D}_{\alpha} \otimes \mathbf{E}^{\alpha} .
$$

The after deformation gradient $\mathbf{F}_{\text {def }}$ will be

$$
\mathbf{F}_{\text {after }}=\mathbf{g}_{i} \otimes \overline{\mathbf{G}}^{i}=\mathbf{r}_{\alpha} \otimes \overline{\mathbf{G}}^{\alpha}+\mathbf{d} \otimes \overline{\mathbf{G}}^{3}+\xi \mathbf{d},_{\alpha} \otimes \overline{\mathbf{G}}^{\alpha} .
$$

According to the superposition of deformation gradient, the total deformation gradient will be

$$
\begin{aligned}
\mathbf{F}_{\text {Total }} & =\mathbf{F}_{\text {def }} \mathbf{F}_{\text {pre }}=\left(\mathbf{g}_{i} \otimes \overline{\mathbf{G}}^{i}\right)\left(\overline{\mathbf{G}}_{i} \otimes \mathbf{G}^{i}\right)=\mathbf{g}_{i} \otimes \mathbf{G}^{i} \\
& =\mathbf{r}_{\alpha} \otimes \mathbf{E}^{\alpha}+\mathbf{d} \otimes \mathbf{E}^{3}+\boldsymbol{\xi} \mathbf{d},_{\alpha} \otimes \mathbf{E}^{\alpha} .
\end{aligned}
$$

The associated Jacobian determinants are given by

$$
J=\operatorname{det}(\mathbf{F})=\frac{j}{j^{0}}, \quad j=\mathbf{g}_{1} \times \mathbf{g}_{2} \cdot \mathbf{g}_{3}, j^{0}=\mathbf{E}_{1} \times \mathbf{E}_{2} \cdot \mathbf{E}_{3},
$$

and from (8) and (9), and if we denote the director field gradient as

$$
\mathbf{d}_{\alpha}=\kappa_{\alpha}^{\beta} \mathbf{a}_{\beta}+\kappa_{\alpha}^{3} \mathbf{d}, \quad \mathbf{D}, \alpha=K_{\alpha}^{\beta} \mathbf{A}_{\alpha}+K_{\alpha}^{3} \mathbf{D} .
$$

then, we have

$$
\begin{aligned}
j=\hat{j}\left(1+2 \xi h+\xi^{2} \kappa\right), & \hat{j}=\left.j\right|_{\xi=0}=\mathbf{r},_{1} \times \mathbf{r}, 2 \cdot \mathbf{d}, \\
\bar{j}=\tilde{j}\left(1+2 \xi H+\xi^{2} K\right), & \tilde{j}=\left.\bar{j}\right|_{\xi=0}=\mathbf{R},,_{1} \times \mathbf{R}, 2 \cdot \mathbf{D},
\end{aligned}
$$

where $h=(1 / 2)\left(\kappa_{1}^{1}+\kappa_{2}^{2}\right), \kappa=\kappa_{1}^{1} \kappa_{2}^{2}-\kappa_{2}^{1} \kappa_{1}^{2}$ can be thought of as the mean and Gaussian curvature in the deformed configuration, and $H=(1 / 2)\left(K_{1}^{1}+K_{2}^{2}\right), K=K_{1}^{1} K_{2}^{2}-K_{1}^{2} K_{2}^{1}$ can be thought of as mean and Gaussian curvature in the pre-deformation configuration. Since no deformation in the

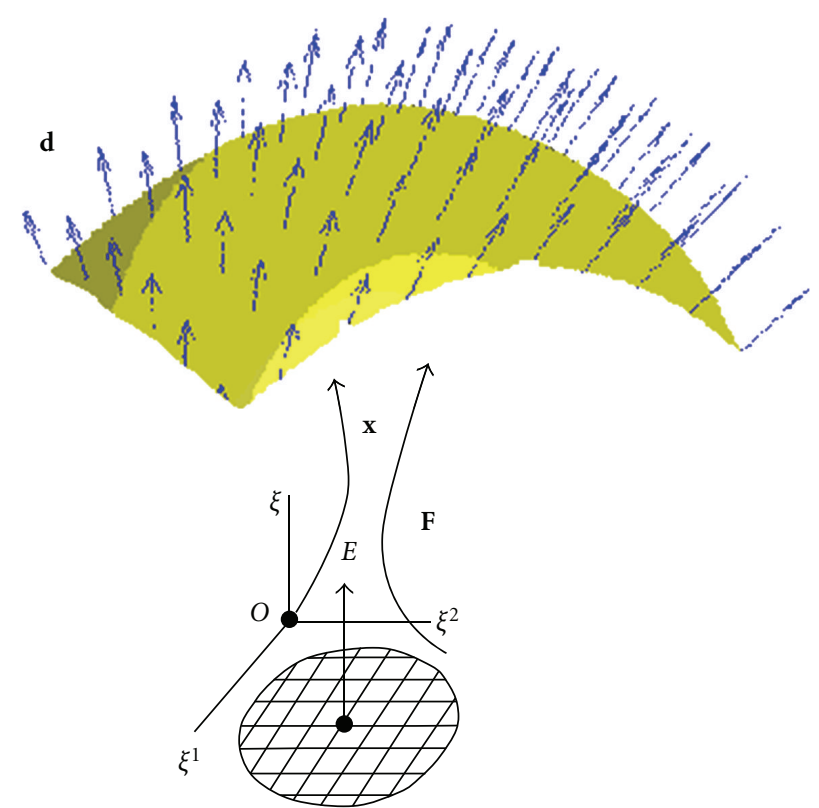

FIGURE 7: The graphene sheet without pre-deformation.

undeformed graphene plane exists, so both its mean and Gaussian curvature are zero.

For instance, the cylindrical pre-deformation [21], $x_{1}=$ $R \sin \left(2 \pi\left(X_{1} / L\right)\right), x_{2}=X_{2}, x_{3}=R-R \cos \left(2 \pi\left(X_{1} / L\right)\right)$, where $L$ is the width of the grapheme sheet before rolling and $R$ is the nanotube radius. The pre-deformation gradient in this case is

$$
\mathbf{F}_{\text {pre }}=\left(\begin{array}{cc}
\frac{2 \pi R}{L} \cos \left(2 \pi \frac{X_{1}}{L}\right) & 0 \\
0 & 1 \\
\frac{2 \pi R}{L} \sin \left(2 \pi \frac{X_{1}}{L}\right) & 0
\end{array}\right) .
$$

\section{Physical Components of Some Quantities in Terms of Chiral Index $(n, m)$}

The chiral index $(n, m)$ is an intrinsic parameter of graphene. With our chiral-tube basis vectors, the influence of the chiral index $(n, m)$ has been introduced into deformation gradient and can be shown explicitly in physical components.

Before going to discuss the physical components of tensor, let us here make a remark about the physical components of physical quantities. We should remember that the tensor (vector) components of a physical quantity which is referred to a particular curvilinear coordinate system may or may not have the same physical dimensions. In this case a great convenience arises because we would like to keep our freedom in choosing arbitrary curvilinear coordinates. For this reason, we must distinguish the tenos components from the "physical components", which must have uniform physical dimensions, but they do not transfer conveniently under coordinate transformations. 


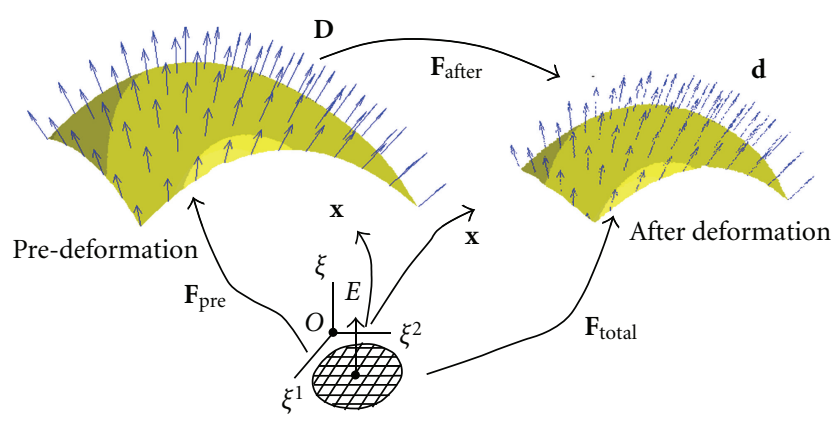

Figure 8: The grapheme with pre-deformation.

First of all, let us consider the physical components of a vector $\mathbf{u}=u^{i} \mathbf{g}_{i}=\mathbf{u}_{i} \mathbf{g}^{i}$. It is clearly that the base vectors $\mathbf{g}_{i}$ and $\mathbf{g}^{i}$ are in general not unit vectors. In fact, their lengths are $\left|\mathbf{g}_{i}\right|=\sqrt{\mathbf{g}_{i} \cdot \mathbf{g}_{i}}=\sqrt{g_{i i}}\left|\mathbf{g}^{i}\right|=\sqrt{\mathbf{g}^{i} \cdot \mathbf{g}^{i}}=\sqrt{g^{i i}}$, i not in summation. Then $\hat{\mathbf{g}}_{i}=\mathbf{g}_{i} / \sqrt{g_{i i}}, \hat{\mathbf{g}}^{i}=\mathbf{g}^{i} / \sqrt{g^{i i}}$ are unit vectors. With the notation of the unit vectors, the vector can be rewriten as

$$
\mathbf{u}=u^{i} \mathbf{g}_{i}=u^{i} \sqrt{g_{i i}} \hat{\mathbf{g}}_{i} \equiv \widehat{u}^{i} \hat{\mathbf{g}}_{i}=u_{i} \mathbf{g}^{i}=u_{i} \sqrt{g^{i i}} \widehat{\mathbf{g}}^{i} \equiv \hat{u}_{i} \hat{\mathbf{g}}^{i},
$$

where $\hat{u}^{i}=u^{i} \sqrt{g_{i i}}, \hat{u}_{i}=u_{i} \sqrt{g^{i i}}$ will have the same physical dimensions. It is seen that $\widehat{u}^{i}$ are the components of $v$ resolved in the direction of unit vectors $\hat{\mathbf{g}}_{i}$ which are tangent to the coordinate lines, and that $\hat{u}^{i}$ are the components of $\mathbf{u}$ resolved in the direction of unit vectors $\hat{\mathbf{g}}^{\mathrm{i}}$ which are perpendicular to the coordinate planes. So the components $\hat{u}^{i}$ and $\hat{u}_{i}$ are called the physical components of the vector $\mathbf{u}$, and $\hat{\mathbf{g}}_{i}$ and $\hat{\mathbf{g}}^{i}$ are physical base vectors. This means that that the physical components of a vector $\mathbf{u}$ can be defined as the components of $\mathbf{u}$ resolved in the directions of a set of unit vectors which are parallel either to the set of base vectors or to the set of reciprocal base vectors.

Similarly, for the reference or undeformed state, then $\widehat{\mathbf{E}}_{i}=\mathbf{E}_{i} / \sqrt{G_{i i}}, \widehat{\mathbf{E}}^{i}=\mathbf{E}^{i} / \sqrt{G^{i i}}$. For tensor calculation, we define the chiral-tube unit physical base vectors as

$$
\hat{\mathbf{E}}_{1}=\frac{\mathbf{C}_{h}}{\left|\mathbf{C}_{h}\right|}, \quad \hat{\mathbf{E}}_{2}=\frac{\mathbf{T}}{|\mathbf{T}|}, \quad \hat{\mathbf{E}}_{3}=\frac{\mathbf{E}_{3}}{\left|\mathbf{E}_{3}\right|},
$$

where $\left|\mathbf{C}_{h}\right|=\sqrt{\mathbf{C}_{h} \cdot \mathbf{C}_{h}}=\sqrt{(n+(\sqrt{3} / 2) m)^{2}+((\sqrt{3} / 2) m)^{2}}$, $|\mathbf{T}|=\sqrt{(n+(\sqrt{3} / 2) m)^{2}+((\sqrt{3} / 2) m)^{2}}$, and $\left|\mathbf{E}_{3}\right|=\left(n^{2}+\right.$ $m \sqrt{3})$. It is indicated in (14) that chiral index has been included into the physical components of base vectors. Since all quantities and variables must be established on the base vectors, all of them will be represented by the chiral index. This is the beauty of this work. For "zigzag" with $m=0$, we have $\hat{\mathbf{E}}_{1}=\mathbf{C}_{h} / n, \hat{\mathbf{E}}_{2}=\mathbf{T} / n, \hat{\mathbf{E}}_{3}=\mathbf{E}_{3} / n^{2}$; for "archair" with $n=m$, we have

$$
\begin{gathered}
\hat{\mathbf{E}}_{1}=\frac{2 \mathbf{C}_{h}}{(n \sqrt{(7+2 \sqrt{3})})}, \quad \hat{\mathbf{E}}_{2}=\frac{2 \mathbf{T}}{n \sqrt{7+2 \sqrt{3}}}, \\
\hat{\mathbf{E}}_{3}=\frac{\mathbf{E}_{3}}{n^{2}(1+\sqrt{3})} .
\end{gathered}
$$

Using the above physical component in (14), for instance, the 2nd Piola-stress tensor of the shell model can be written in physical component form:

$$
\begin{aligned}
\mathbf{S} & =J \mathbf{F}^{-1} \boldsymbol{\sigma} \mathbf{F}^{-T}=J \sigma^{i j} \mathbf{E}_{i} \otimes \mathbf{E}_{j} \\
& =J \sigma^{i j} \sqrt{E_{i i}} \sqrt{G_{j j}} \widehat{\mathbf{E}}_{i} \otimes \widehat{\mathbf{E}}_{j} \equiv \widehat{S}^{i j} \widehat{\mathbf{E}}_{i} \otimes \widehat{\mathbf{E}}_{j} .
\end{aligned}
$$

Similarly, all other quantities can be explicitly expressed in terms of the chiral index $(n, m)$.

\section{Conclusion}

This paper investigates the possibility of introducing the chiral index $(n, m)$ into the deformation formulation of graphene continuum shell model. For this purpose, we established a chiral-tube basis vectors from graphene unit vectors in real space and formulated graphene deformation gradient for both with and without predeformation. The importance of the chiral index on the deformation and stress has been shown explicitly in the physical components.

\section{Acknowledgments}

This work was supported by South Africa National Research Foundation. This support is gratefully acknowledged. The author would like to express his thankfulness to the constructive comments and insightful suggestions given by three anonymous referees.

\section{References}

[1] A. K. Geim and K. S. Novoselov, "The rise of graphene," Nature Materials, vol. 6, no. 3, pp. 183-191, 2007.

[2] A. K. Geim, "Graphene: status and prospects," Science, vol. 324, no. 5934, pp. 1530-1534, 2009.

[3] A. K. Geim and K. S. Novoselov, "The rise of graphene," Nature Materials, vol. 6, no. 3, pp. 183-191, 2007.

[4] B. Sun and X. M. Henry Huang, "Mechanical nano-resonators at ultra-high frequency and their potential applications," South Africa Journal of Science, vol. 104, pp. 169-171, 2008.

[5] C. Q. Ru, "Axially compressed buckling of a doublewalled carbon nanotube embedded in an elastic medium," Journal of the Mechanics and Physics of Solids, vol. 49, no. 6, pp. 12651279, 2001.

[6] Y. Wu, M. Huang, F. Wang et al., "Determination of the young's modulus of structurally defined carbon nanotubes," Nano Letters, vol. 8, no. 12, pp. 4158-4161, 2008.

[7] B. Sun, "Deformation, vibration, and buckling of nanotorus," Journal of Nanomaterials. In press.

[8] W. Z. Chien, "The instrisic theory of thin shells and plates, I," Quarterly of Applied Mathematics, vol. 1, pp. 297-327, 1943.

[9] W. Z. Chien, "The instrisic theory of thin shells and plates, II," Quarterly of Applied Mathematics, vol. 2, pp. 120-135, 1944.

[10] W. T. Koiter, "On the nonlinear theory of thin elastic shells," Proceedings of the Koninklijke Nederlandse Akademie van Wetenschappen. Series B, vol. 69, pp. 1-54, 1966.

[11] P. M. Naghdi, The Theory of Shells, Handbuch der Physik, vol. 2, Springer, Berlin, Germany, 1972, edited by C. Truesdell.

[12] J. L. Sanders, "Non-linear theories for thin shells," Quarterly of Applied Mathematics, vol. 21, pp. 21-36, 1963. 
[13] J. C. Simo and D. D. Fox, "On a stress resultant geometrically exact shell model. Part I: formulation and optimal parametrization," Computer Methods in Applied Mechanics and Engineering, vol. 72, no. 3, pp. 267-304, 1989.

[14] B. Sun and R. Liu, "Single director shell model without complex geometric concepts," Advance in Mechanics, vol. 35, no. 2, pp. 181-194, 2003.

[15] C. Lee, X. Wei, J. W. Kysar, and J. Hone, "Measurement of the elastic properties and intrinsic strength of monolayer graphene," Science, vol. 321, no. 5887, pp. 385-388, 2008.

[16] J. Atalaya, A. Isacsson, and J. M. Kinaret, "Continuum elastic modeling of graphene resonators," Nano Letters, vol. 8, no. 12, pp. 4196-4200, 2008.

[17] J. S. Bunch, S. S. Verbridge, J. S. Alden et al., "Impermeable atomic membranes from graphene sheets," Nano Letters, vol. 8, no. 8, pp. 2458-2462, 2008.

[18] B. I. Yakobson, C. J. Brabec, and J. Bernholc, "Nanomechanics of carbon tubes: instabilities beyond linear response," Physical Review Letters, vol. 76, pp. 2511-2514, 1996.

[19] M. Arroyo and T. Belytschko, "Finite crystal elasticity of carbon nanotubes based on the exponential Cauchy-Born rule," Physical Review B, vol. 69, no. 11, Article ID 115415, 11 pages, 2004.

[20] Y. Huang, J. Wu, and K. C. Hwang, "Thickness of graphene and single-wall carbon nanotubes," Physical Review B, vol. 74, no. 24, Article ID 245413, 2006.

[21] Q. Lu and R. Huang, "Nonlinear mechanics of singleatom-layer graphene sheets," International Journal of Applied Mechanics, vol. 1, no. 3, pp. 443-467, 2009.

[22] Z.-Q. Wang, Y.-P. Zhao, and Z.-P. Huang, "The effects of surface tension on the elastic properties of nano structures," International Journal of Engineering Science, vol. 48, no. 2, pp. 140-150, 2010. 

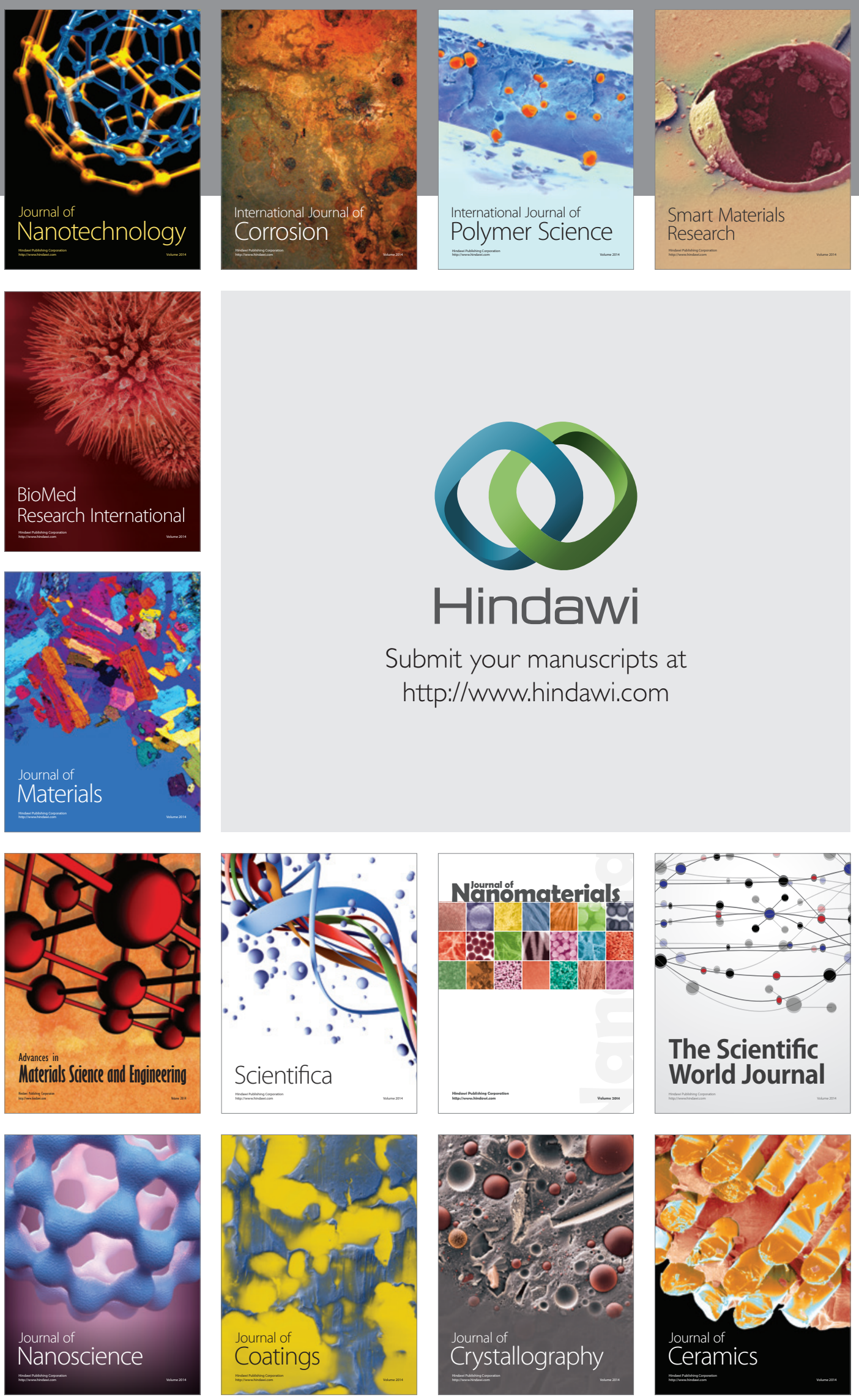

The Scientific World Journal

Submit your manuscripts at

http://www.hindawi.com

\section{World Journal}

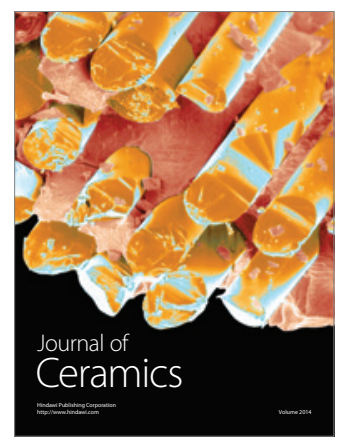

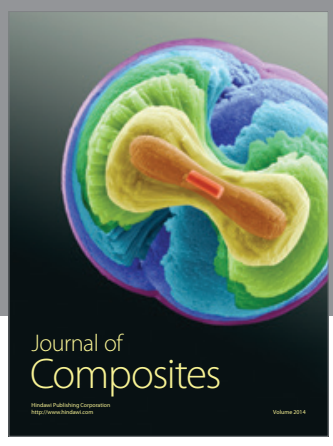
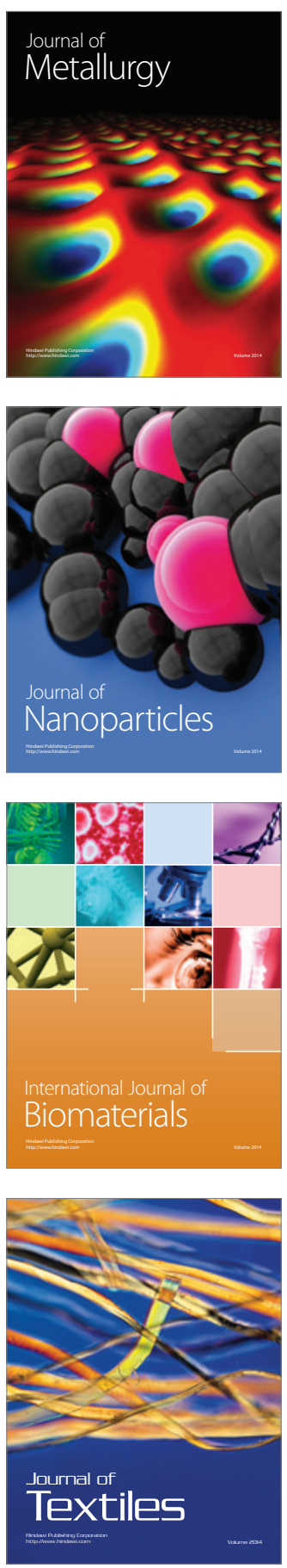